\title{
GPU-based Simulations of Fracture in Idealized Brick and Mortar Composites
}

\author{
J. William Pro \\ Department of Mechanical Engineering, 2235C Engineering II Building, University of California, Santa Barbara, \\ CA 93106, USA \\ Rone Kwei Lim \\ Department of Computer Science, 5110 Harold Frank Hall, University of California, Santa Barbara, CA 93106, USA \\ Linda R. Petzold \\ Department of Mechanical Engineering and Department of Computer Science, 5107 Harold Frank Hall, University \\ of California, Santa Barbara, CA 93106, USA \\ Marcel Utz \\ School of Chemistry, University of Southampton, SO17 1BJ, UK \\ Matthew R. Begley ${ }^{1}$ \\ Materials Department and Department of Mechanical Engineering, 2361A Engineering II Building, University of \\ California, Santa Barbara, CA 93106, USA
}

\begin{abstract}
Stiff ceramic platelets (or bricks) that are aligned and bonded to a second ductile phase with low volume fraction (mortar) are a promising pathway to produce stiff, high-toughness composites. For certain ranges of constituent properties, including those of some synthetic analogues to nacre, one can demonstrate that the deformation is dominated by relative brick motions. This paper describes simulations of fracture that explicitly track the motions of individual rigid bricks in an idealized microstructure; cohesive tractions acting between the bricks introduce elastic, plastic and rupture behaviors. Results are presented for the stresses and damage near macroscopic cracks with different brick orientations relative to the loading orientation. The anisotropic macroscopic initiation toughness is computed for small-scale yielding conditions and is shown to be independent of specimen geometry and loading configuration. The results are shown to be in agreement with previously published experiments on synthetic nacre.
\end{abstract}

\footnotetext{
${ }^{1}$ begley@engineering.ucsb.edu, fax:(805) 893-8486
} 


\section{Introduction}

Composite materials consisting of aligned stiff platelets (or whiskers) bonded together with low volume fractions of a ductile phase are a promising pathway to produce high-performance macroscopic properties. A well-known example is nacre, commonly found on the inner layer of certain shelled species (e.g., snails, mollusks, etc.); its highly intricate, hierarchical microstructure leads to performance that greatly exceeds 'rule of mixtures' estimates based on its mostly brittle composition (Wegst and Ashby, 2004; Meyers et al., 2008; Barthelat et al., 2007). Similar to other natural composites (e.g. antler and bone), nacre has inspired many studies with the hope of developing synthetic analogues with improved performance (Currey, 1977; Jackson et al., 1990; Kamat et al., 2000; Evans et al., 2001; Wang et al., 2001; Okumura and De Gennes, 2001; Menig et al., 2001; Vincent, 2003; Bouville et al., 2014; Deville et al., 2006). In essence, all of these microstructures consist of an overlapping 'brick and mortar' structure shown schematically in Figure 1. For natural nacre, the bricks are an idealization of small tablets of brittle calcium carbonate (aragonite), comprising nearly $95-99 \%$ of the microstructure volume fraction; the mortar is an idealization of a thin, organic layer of biopolymer (1-5\% volume fraction). The resulting macroscopic toughness is approximately $300 \mathrm{~J} / \mathrm{m}^{2}$, nearly thirty times higher (in energy terms) than a bulk aragonite monolith (Barthelat and Espinosa, 2007), an amplification yet to be reproduced by any man-made replica (Wegst and Ashby, 2004; Barthelat, 2007).

While nacre is an inspiring example of a 'brick and mortar' microstructure, the goal of the present work is to explore the implications of microstructural features for synthetic analogues. For this reason, certain important phenomena of nacre, such as asperity sliding (Katti et al., 2004; Evans et al., 2001) and bridging ligaments formed from the brittle phase (Meyers et al., 2008; Song et al., 2003), are not considered here. Over the past decade, advanced processing techniques have increased the potential for cost effective and efficient routes to processing nacre-like structures in bulk form (Finnemore et al., 2012; Launey et al., 2009). Analogous to natural nacre, current synthetics utilize a large volume fraction of a hard, brittle material to promote strength along with a ductile polymer to promote toughness. A notable example is the composite formed with alumina $\left(\mathrm{Al}_{2} \mathrm{O}_{3}\right)$ as the brittle 'brick' phase and poly-methyl-methacrylate (PMMA) as the ductile 'mortar' phase (Munch et al., 2008). Fundamental questions remain regarding how the microstructure (e.g. 


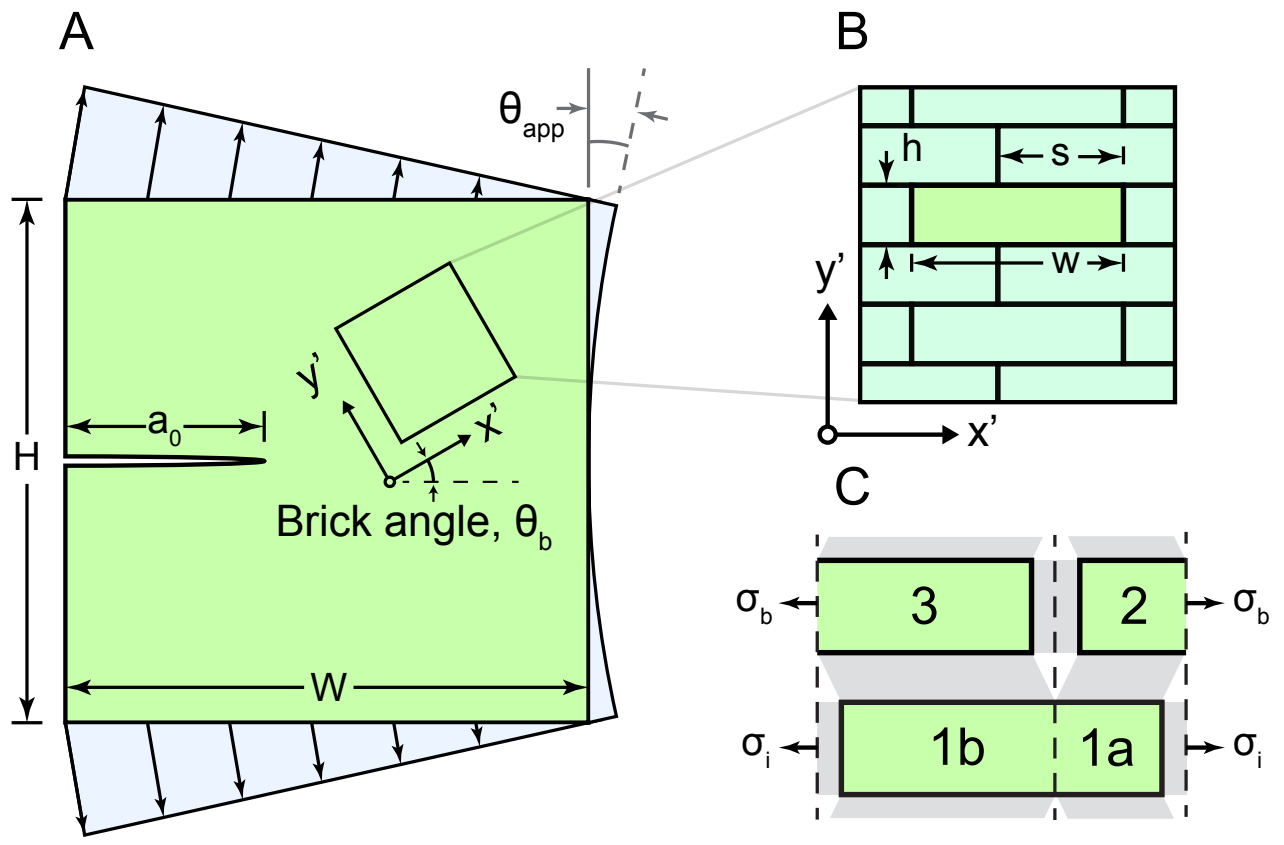

Figure 1: Schematic of (a) macroscopic specimen and loading (b) brick parameters and (c) unit cell used in analysis.

brick aspect ratio, overlap, mortar volume fraction, etc.) and constituent properties (e.g. brick strength, mortar yield and rupture strains) should be tailored to optimize composite properties for these synthetics.

The strength and stiffness of 'brick and mortar' composites have been extensively modeled analytically for homogeneous deformation, predominantly for loading in the long brick direction (Begley et al., 2012; Wilbrink et al., 2010; Kotha et al., 2001; Jäger and Fratzl, 2000; Bertoldi et al., 2008; Barthelat and Rabiei, 2011; Jackson et al., 1988; Genet et al., 2014), as shown in Figure 1C. These works provide key insights into the impact of microstructural properties on macroscopic performance. However, the response of these materials to non-uniform stress and deformation is much less characterized, resulting in a less established quantitative understanding of the link between microstructure and the resulting anisotropy, failure mechanisms, and macroscopic toughness. In this regard, work by Rabiei et al. (2010) offers insight into the various failure mechanisms of brick-andmortar structures along with the associated material strength under multiple loading orientations. In separate work, the same authors developed an analytical model that accurately captures the toughening behavior for a loading orientation parallel to the long brick direction (Barthelat and Rabiei, 2011). However, there are currently no predictive models for the fracture initiation tough- 
ness in terms of the complex anisotropy of these structures, or the transitions between associated failure mechanisms. This is largely due to the relative computational cost of fracture simulations with different length scales for the process zone size, the brick size, and the specimen size.

The aim of the present work is to present an idealized, efficient model that captures the interplay between the material anisotropy, loading direction, and initiation toughness. To simplify the analysis and reduce the computational cost of the problem, the model idealizes the bricks as rigid and represents the mortar using cohesive laws. These significant assumptions are naturally only valid for a certain range of constituent properties, which imply the mortar compliance (as dictated by its volume fraction and modulus) is far greater than that of the bricks. In a subsequent section, scaling laws based on previous micromechanical models are used to explicitly quantify the range of properties for which this approximation is valid. The idealization implies that the deformation in the material is completely described by the motion of the bricks, which interact only with their nearest neighbors through cohesive laws. As a result, highly efficient and stable computational schemes can be parallelized to a significant degree; this enables large-scale simulations involving damage zones spanning thousands of bricks. Hence, the approach captures the non-uniform stress states ahead of a dominant flaw as well as growth of the damage zone with high resolution. In turn, this offers new insight into the effect of microstructure on the overall macroscopic composite properties.

The present approach builds upon the many insights into nacreous microstructures generated previously using 2D and 3D finite element approaches, many of which incorporate cohesive laws similar those described here. These previous cohesive models have identified key aspects of the influence of microstructure on the composite stress-strain response (Bekah et al., 2012; Tang et al., 2007; Barthelat et al., 2007) and crack propagation (An et al., 2014; Flores-Johnson et al., 2014; Nguyen and Govindjee, 2006). The work presented here confirms many similar behaviors, and extends them by developing an efficient numerical framework that enables extraction of the fracture initiation toughness as a function of orientation and constituent properties. The efficiency of the current framework is an important advance, as it enables systematic studies of features not previously considered, such as stochastic brick sizes and overlap (Zhang et al., 2010a; Lei et al., 2012) and hierarchical brick arrangements (Zhang et al., 2010b). 


\section{Material model}

Figure 1B illustrates the idealized microstructure utilized in the present simulations; an overlapping array of comparatively stiff bricks is bonded by thin, compliant mortar sections. We approximate the bricks as rigid (justified next) and implicitly account for the behavior of the mortar using cohesive laws that dictate the tractions between bricks and the local rupture properties. The mortar exhibits elastic perfectly-plastic behavior up to a critical rupture strain. The mortar thickness is treated as negligible in comparison to the brick dimensions, and appears only implicitly through the cohesive law. That is, the rupture strain for the mortar is implicitly defined as a critical separation between bricks divided by the mortar thickness.

The present simulations involve bricks of equal size, although the formulation is general in the sense it can handle arbitrary brick shapes and size distributions. The influence of statistical distributions in brick sizes is examined in a companion paper by Pro et al. (2015). In the present simulations, we neglect the possibility of brick failures, although the formulation could be adapted to account for this possibility by utilizing 'bricks within bricks', with internal cohesive laws that are different from those describing the interaction between adjacent bricks. Given the significance of the rigid brick approximation, we first detail in Section 2.1 the combinations of brick and mortar properties for which the approximation is valid, using a micromechanical model for tension in the brick direction shown in Figure 1C. The constitutive description of the mortar is then described in Section 2.2. The governing equations and solution technique is described in Section 3.

\subsection{Rigid brick approximation}

Begley et al. (2012) described a micromechanical model that accounts for brick elongation when the composite is subjected to axial tension, which identifies the key dimensionless parameters that define whether the rigid brick assumption is valid. Loading in the long brick direction produces the most stringent limitations in system properties for the rigid brick approximation; in this orientation, shear transfer between overlapping bricks leads to largest possible stresses that act to elongate the bricks. The uniaxial response of the composite shown in Figure $1 \mathrm{C}$ is analyzed assuming that (i) the horizontal mortar sections experience pure shear and the vertical mortar sections experience pure tension, and (ii) the bricks experience purely axial elongation. The resulting model yields an analytical solution for the displacement distributions in the bricks, which are entirely described by 
the following two dimensionless parameters:

$$
\kappa_{1}=\frac{\bar{E}_{m} w}{\bar{E}_{b} t} ; \quad \kappa_{2}=\sqrt{\frac{\left(1-v_{m}\right) \bar{E}_{m} w^{2}}{\bar{E}_{b} h t}}
$$

where $\bar{E}_{m}=E_{m} /\left(1-v_{m}^{2}\right)$ is the plane strain modulus of the mortar (with $\bar{E}_{b}$ defined equivalently for the bricks), $t$ is the mortar thickness, $h$ is the brick height, and $w$ is the brick width. Here, the displacement fields from the Begley et al. (2012) model are used to derive the elastic energy in the composite in terms of $\kappa_{1,2}$; the rigid brick approximation (with $\kappa_{1,2} \approx 0$ ) neglects energetic contributions on the order of $\kappa_{1,2}$ compared to unity.

In what follows, $\bar{u}_{i}(x)=u_{i}(x) / w$ are the normalized displacement distributions in the bricks, with $i$ indicating the relevant brick (see Figure 1C). For simplicity, we consider the case where the brick overlap is exactly one-half the brick width, i.e. $s=w / 2$. The strain energy in the bricks is given by:

$$
\Pi_{b}=2 h \int_{0}^{w / 2} \frac{1}{2} \frac{\sigma_{b}^{2}}{\bar{E}_{b}} d x=h w \bar{E}_{b} \int_{0}^{1 / 2}\left(\bar{u}_{1}^{\prime}\right)^{2} d \bar{x}=\bar{E}_{b} h w \epsilon_{o}^{2} f_{b}\left(\kappa_{1}, \kappa_{2}\right)
$$

where $\epsilon_{o}$ is the macroscopic applied strain imposed on the unit cell, $\bar{u}_{1}$ is the displacement distribution in one-half of the brick, and the prime denotes differentiation with respect to $\bar{x}=x / w$. The dimensionless function $f_{b}\left(\kappa_{1}, \kappa_{2}\right)$ can be directly obtained from the displacement solution in Begley et al. (2012). The strain energy in the horizontal mortar sections (between brick ends) is given by:

$$
\Pi_{m}^{s}=2 t \int_{0}^{w / 2} \frac{1}{2} \tau_{i} \gamma_{i} d x=t w G_{m} \int_{0}^{1 / 2}\left[\frac{w}{t}\left(\bar{u}_{2}-\bar{u}_{1 a}\right)\right]^{2} d \bar{x}=\frac{\left(1-v_{m}\right) \bar{E}_{m} w^{3}}{2 t} \epsilon_{o}^{2} f^{s}\left(\kappa_{1}, \kappa_{2}\right)
$$

where $f^{s}\left(\kappa_{1}, \kappa_{2}\right)$ is another dimensionless function that can be recovered using the solution in Begley et al. (2012) and performing the above integral. The strain energy in the vertical mortar sections is given by:

$$
\begin{aligned}
\Pi_{m}^{t} & =t h \cdot \frac{1}{2} \bar{E}_{m}\left(\frac{2 u_{2}(0)}{t}\right)^{2}=\frac{2 h w^{2}}{t} \bar{E}_{m} \bar{u}_{2}(0)^{2} \\
& =\frac{2 w^{2} h}{t} \bar{E}_{m} \epsilon_{o}^{2} f_{b}^{t}\left(\kappa_{1}, \kappa_{2}\right)
\end{aligned}
$$

where again, $f_{b}^{t}$ is a dimensionless function dictated by the displacement solution. Thus, the total strain energy in the system can be written as:

$$
\frac{\Pi_{t o t}}{\bar{E}_{m} w h \epsilon_{o}^{2}}=\frac{\left(1-v_{m}\right) \bar{w}^{2}}{2 h t}\left(\frac{2}{\kappa_{2}^{2}} f_{b}\left(\kappa_{1}, \kappa_{2}\right)+f^{s}\left(\kappa_{1}, \kappa_{2}\right)+\alpha f_{b}^{t}\left(\kappa_{1}, \kappa_{2}\right)\right)
$$


where $\alpha=4 h /\left[\left(1-v_{m}\right) w\right]$.

The rigid brick approximation assumes $\kappa_{1,2} \approx 0$, as implied by eqn. (1) above and $\bar{E}_{b} \rightarrow \infty$. A series expansion of the above functions about $\left(\kappa_{1}=0, \kappa_{2}=0\right)$ produces:

$$
\frac{\Pi_{t o t}}{\bar{E}_{m} w h \epsilon_{o}^{2}}=\frac{\left(1-v_{m}\right) \bar{w}^{2}}{2 h t}\left[\frac{1}{8}+\frac{\alpha}{4}-\alpha \frac{\kappa_{1}}{2}-\frac{\kappa_{2}^{2}}{8}\left(\frac{1}{3}+\alpha\right)\right]
$$

where it should be noted that $O\left[\kappa_{1}\right]=O\left[\kappa_{2}^{2}\right]$, such that second order terms on $\kappa_{2}$ should be retained. The rigid brick approximation retains the first two terms and neglects the remainder: the above solution is easily confirmed by assuming a priori that the bricks are rigid and solving for the energy in the mortar.

Note that if one uses the effective composite modulus defined as $\bar{E}_{c}\left(\kappa_{1}, \kappa_{2}\right)$ in Begley et al. (2012) to compute the strain energy in the composite via $\Pi_{t o t}=(1 / 2) \bar{E}_{c} \epsilon_{o}^{2}$, one obtains identical scaling, only with slightly different coefficients to the $\kappa$ terms (that are different functions of $\alpha$ ). The slight discrepancy between pre-factors in eqn. (7) and those obtained from the modulus definition in Begley et al. (2012) arises due to the fact that the mortar is assumed infinitesimal in comparison to other dimensions. This condition is not imposed in the above derivation. It is also worth noting that if one computes the average composite stress, i.e. $\sigma_{c}=\bar{E}_{c} \epsilon_{o}$, one determines that the errors in composite stress associated with the rigid brick assumption are controlled by identical scaling factors.

The error terms (indicated by the $\kappa$ terms above, which are neglected in the rigid brick approximation) are negative, indicating that the energy and average composite stress in the rigid brick model are overestimates of those obtained when the bricks are deformable. This is a consequence of the fact that allowing for brick deformation for a given level of imposed strain would lower the energy by increasing the compliance. The opposite is true if tractions are prescribed: under those conditions, the strain energy and stresses in the rigid brick model would be underestimates.

Using the above results, one can define the fractional error in energy associated with the rigid brick approximation as:

$$
\frac{\Delta \Pi}{\Pi}=\frac{4 \alpha}{1+2 \alpha} \kappa_{1}+\frac{1+3 \alpha}{3(1+2 \alpha)} \kappa_{2}^{2}
$$

This estimate in the error associated with the rigid brick approximation is illustrated in Figure 2 , which depicts contours of the error as a function of modulus ratio $\left(\bar{E}_{m} / \bar{E}_{b}\right)$ and brick aspect ratio $(\mathrm{w} / \mathrm{h})$. In this plot, the thickness of the mortar $(\mathrm{t})$ is replaced with a suitable expression 
involving the mortar volume fraction, taken here to be $\mathrm{f}=0.1$. It is clear from the figure that the present model only applies for modest aspect ratios, extremely compliant mortars (relative to that of the brick), and volume fractions much larger than those seen for natural materials. However, for synthetic materials such as those in Launey et al. (2009) and Munch et al. (2008), the present simulations would have errors on the order of $10-20 \%$, which is reasonable in light of the considerable reduction in complexity associated with the rigid brick approximation.

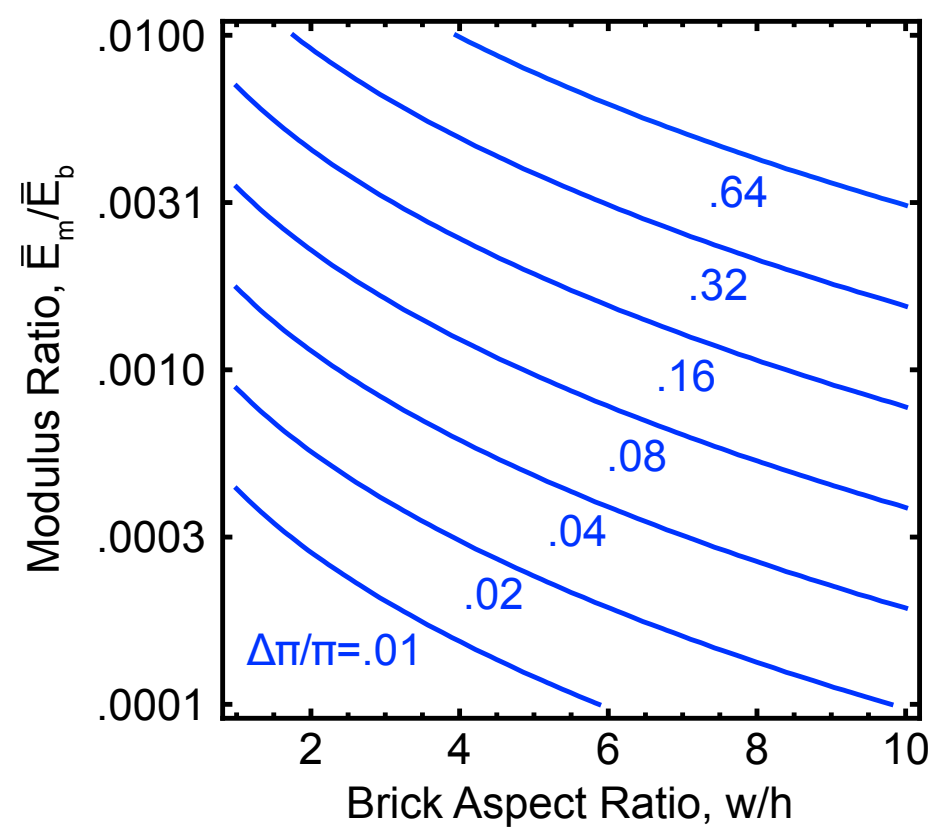

Figure 2: Contours of fractional error in energy associated with the rigid brick assumption.

Additionally, in order to estimate the error in computed fracture toughness due to the rigid brick approximation (in terms of the fractional error in energy), a set of supplementary simulations was also performed using deformable, constant strain triangular elements. To limit the scope while providing a qualitative comparison, each brick was split on the diagonals to create four triangular elements. The calculations showed that, in the limit that $\kappa_{1,2} \rightarrow 0$, one recovers the result for fracture toughness computed in the rigid brick simulations to within a few percent. As compliance is introduced into the bricks, thereby increasing the fractional error in energy relative to the rigid simulations, the fractional error in the computed toughness also increases. Though the deformable brick simulations were not fully resolved (i.e. with a larger number of constant strain triangles in each brick), the fracture toughness from the rigid brick simulations was less than $5 \%$ higher than 
the deformable brick simulations when considering fractional energy errors less than about 0.1. Therefore, the overestimation of energy from the rigid brick simulations can be higher than the overestimate in toughness. Thus, it can be inferred that the rigid brick simulations presented here overestimate the fracture toughness as compared to simulations with deformable bricks, likely with smaller errors than those associated with elastic strain energy described above.

\subsection{Mortar properties: cohesive laws describing brick interactions}

The constitutive description of the mortar material defines the cohesive tractions holding bricks together, and the work required to rupture the interface between adjacent bricks. Here, we adopt a phenomenological elastic perfectly-plastic description for the mortar: other constitutive descriptions can be substituted without changes to what follows. Rupture is modeled by specifying that the mortar does not support tractions once a threshold rupture strain is exceeded, as shown in Figure 3. The mathematical description is given by:

$$
T_{n}= \begin{cases}k_{n} \Delta_{n} & \text { if } \sqrt{\Delta_{n}^{2}+\Delta_{t}^{2}}<\Delta_{Y} \\ \frac{k_{n} \Delta_{Y} \Delta_{n}}{\sqrt{\Delta_{n}^{2}+\Delta_{t}^{2}}} & \text { if } \Delta_{Y} \leq \sqrt{\Delta_{n}^{2}+\Delta_{t}^{2}} \leq \Delta_{R} \\ -k_{n} \Delta_{n}\left(1-\frac{\Delta_{Y}+\Delta_{R}}{\sqrt{\Delta_{n}^{2}+\Delta_{t}^{2}}}\right) & \text { if } \Delta_{R} \leq \sqrt{\Delta_{n}^{2}+\Delta_{t}^{2}} \leq \Delta_{Y}+\Delta_{R} \\ 0 & \text { if } \sqrt{\Delta_{n}^{2}+\Delta_{t}^{2}}>\Delta_{Y}+\Delta_{R}\end{cases}
$$

$+$

$$
\begin{cases}\frac{6 k_{n} \Delta_{n}^{5}}{16 \Delta_{Y}^{4}} & \text { if } \Delta_{n} \leq 0 \\ 0 & \text { if } \Delta_{n}>0\end{cases}
$$

where $T_{n}$ is the normal traction acting on the bricks forming an interface, $\Delta_{n}$ is the normal separation between adjacent bricks, $\Delta_{t}$ is the sliding separation between bricks, $\Delta_{Y}$ is the separation at which yielding occurs, and $\Delta_{R}$ is the separation at which rupture occurs. The shear traction acting between two adjacent bricks is given by:

$$
T_{t}= \begin{cases}k_{n} \Delta_{t} & \text { if } \sqrt{\Delta_{n}^{2}+\Delta_{t}^{2}}<\Delta_{Y} \\ \frac{k_{n} \Delta_{Y} \Delta_{t}}{\sqrt{\Delta_{n}^{2}+\Delta_{t}^{2}}} & \text { if } \Delta_{Y} \leq \sqrt{\Delta_{n}^{2}+\Delta_{t}^{2}} \leq \Delta_{R} \\ -k_{n} \Delta_{t}\left(1-\frac{\Delta_{Y}+\Delta_{R}}{\sqrt{\Delta_{n}^{2}+\Delta_{t}^{2}}}\right) & \text { if } \Delta_{R} \leq \sqrt{\Delta_{n}^{2}+\Delta_{t}^{2}} \leq \Delta_{Y}+\Delta_{R} \\ 0 & \text { if } \sqrt{\Delta_{n}^{2}+\Delta_{t}^{2}}>\Delta_{Y}+\Delta_{R}\end{cases}
$$


The mortar thickness is implicitly accounted for by defining $\Delta_{n}=t \epsilon$ (with $\epsilon$ being the direct strain in the mortar) and $\Delta_{t}=t \gamma$ (with $\gamma$ being the shear strain in the mortar). The (elastic) stiffness of the interfaces are $k_{n}=\bar{E}_{m} / t$. (Naturally, one could define different elastic interface stiffness values for shear and normal separations). As illustrated in Figure 3, the normal and tangential behaviors are coupled, such that normal separation will alter the tangential tractions that can be maintained, and vice versa.
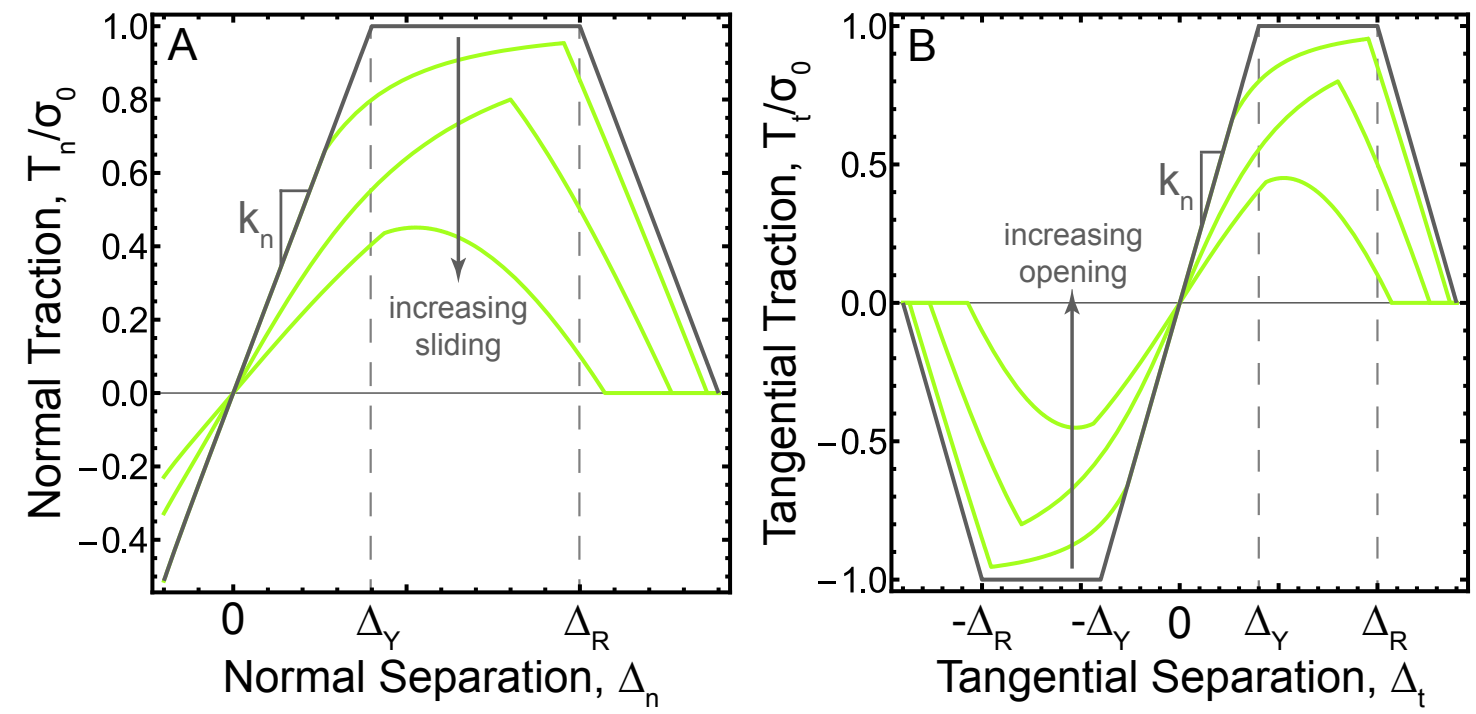

Figure 3: Schematic of the piecewise cohesive law used in analysis for (a) normal and (b) tangential tractions.

The above traction-displacement relationships are used to describe a non-linear elastic material, such that an efficient energy minimization approach can be taken to solve for the deformation. Naturally, this leads to the non-physical behavior that interfaces will 'heal' if they experience unloading prior to rupture. First and foremost, it should be emphasized that the present manuscript applies only to crack initiation, not crack propagation. That is, the present manuscript considers only behaviors that occur under monotonic loading of a pre-defined crack up until the point that the pre-crack just begins to propagate. After initiation, when the crack begins to propagate, unloading effects in the damage zone are important and potentially lead to R-curve behavior, as observed in nacreous materials and their synthetic analogues. As the present formulation does not properly treat unloading (because it allows for full elastic recovery of damaged yet unloading interfaces), it is strictly not suitable to solving for the R-curve behavior. Hence, we focus on predictions 
only of the initiation toughness: this can be justified, even for a material that elastically recovers, simply because unloading effects are rather small during monotonic loading prior to crack growth (McMeeking, 1977). One way of considering the present simulations is that they apply deformation plasticity theory to compute the level of applied load required to reach a critical opening (rupture strain) at the tip of a monotonically loaded pre-crack. To properly simulate R-curve behavior, the present model would have to be adapted to account for energy dissipation that occurs when the mortar is damaged but not yet ruptured. The corresponding form of the cohesive law is given by:

$$
\phi\left(\Delta_{n}, \Delta_{t}\right)=g\left(\Delta_{n}\right)+f\left(\sqrt{\Delta_{t}^{2}+\Delta_{n}^{2}}\right)
$$

where the piece-wise functions $\mathrm{g}(\xi)$ and $\mathrm{f}(\xi)$ are given by:

$$
\begin{gathered}
f(\xi)= \begin{cases}\frac{1}{2} k_{n} \xi^{2} & \text { if } \xi<\Delta_{Y} \\
k_{n} \Delta_{Y}\left(\xi-\frac{1}{2} \Delta_{Y}\right) & \text { if } \Delta_{Y} \leq \xi \leq \Delta_{R} \\
-\frac{1}{2} k_{n}\left[\Delta_{Y}^{2}+\Delta_{R}^{2}+\xi^{2}-2 \xi\left(\Delta_{Y}+\Delta_{R}\right)\right] & \text { if } \Delta_{R} \leq \xi \leq \Delta_{Y}+\Delta_{R} \\
k_{n} \Delta_{Y} \Delta_{R} & \text { if } \xi>\Delta_{Y}+\Delta_{R}\end{cases} \\
g(\xi)= \begin{cases}\frac{k_{n} \xi^{6}}{\Delta_{Y}^{4}} & \text { if } \xi \leq 0 \\
0 & \text { if } \xi>0\end{cases}
\end{gathered}
$$

This potential function yields the above cohesive laws through the definitions $T_{n}=\partial \phi / \partial \Delta_{n}$ and $T_{t}=\partial \phi / \partial \Delta_{t}$. Note that the work of separation $\Gamma_{i}$ is controlled by the parameters $k_{n}$ (the interface stiffness), $\Delta_{Y}$ (the elastic limit of the interface) and $\Delta_{R}$ (the critical displacement for rupture). The work of separation of the interfaces, which is defined here as the intrinsic toughness of the material, is given by:

$$
\Gamma_{i}=\int_{0}^{\infty} T_{n}\left(\Delta_{n}, 0, \Delta_{Y}, \Delta_{R}, k_{n}\right) d \Delta_{n}=k_{n} \Delta_{Y} \Delta_{R}
$$

The objective of the present work is to determine the macroscopic toughness of the bulk material relative to this interface toughness, as a function of brick aspect ratio and loading orientation. The extraction of macroscopic toughness via simulations is described in the next section. 


\section{Governing equations and simulation approach}

\subsection{Governing equations}

For any non-linear elastic material, the global energy minima correspond to the different states of static equilibrium; here, this implies that solutions are defined by finding the brick positions and rotations that minimize the potential energy contained in the cohesive zones. Since the bricks are treated as rigid, the only kinematic expressions needed for the formulation are those that translate global brick positions and orientations into the brick face separations. That is, for neighboring bricks $i$ and $j$, the interface separations are computed as:

$$
\Delta_{n}(s)=f_{n}\left(\underline{x}_{i}, \theta_{i}, \underline{x}_{j}, \theta_{j}\right) ; \quad \Delta_{t}(s)=f_{t}\left(\underline{x}_{i}, \theta_{i}, \underline{x}_{j}, \theta_{j}\right)
$$

where $\underline{x}$ is the position of the brick, $\theta$ is the global orientation of the element, and $s$ is a coordinate along the interface that is determined from the shape of the brick and the global position. The functions $f_{t, n}$ are easily defined using vector mechanics. The system's energy is computed by numerically integrating the cohesive potential given above along all interfaces in the model:

$$
\Phi\left[\left\{\underline{x}_{N}\right\},\left\{\theta_{N}\right\}\right]=\sum_{k}^{M} \int_{S} \phi\left[\Delta_{n}(s), \Delta_{t}(s)\right] d S
$$

where $\left\{\underline{x}_{N}\right\}$ is a vector list of all brick positions, $\left\{\theta_{N}\right\}$ is a vector list of all brick rotations, with $N$ being the number of bricks, and $M$ being the number of interfaces. The number of global degrees of freedom is $3 N$, and the number of energy contributions is $M$. Note that overlapping bricks create multiple interface segments, with openings that are defined by different sets of bricks: each of these interface segments are addressed individually. In the present simulations, numerical integration is used and is based on the opening displacements at the ends of the interface segment being addressed. In this paper, we consider only loading scenarios corresponding to displacement control: for applied tractions, the potential energy of the system should be amended to include contributions due to the work done by applied forces.

\subsection{Monte Carlo minimization}

While non-linear equilibrium equations can be derived from the above energy potential (corresponding to the sum of forces and moments on each brick) and solved using traditional methods, a direct search algorithm is adapted here to minimize the total system energy. The method has 
two significant advantages: first, it is highly robust and insensitive to large discrepancies in local stiffness associated with the emergence of cracks. Second, the 'nearest neighbor' connectivity of the system allows for parallel energy computations. The model and solution method are similar to Monte Carlo strategies commonly used in atomistic simulations (Allen et al., 1987). These methods are well established in many areas of physical modeling as a means to minimize energies and to explore ensemble properties (Binder and Heermann, 2010) and can be generalized for higher dimensional functions of continuous variables (Corana et al., 1987). In essence, the technique involves moving a given brick while holding the neighbors fixed, and computing the associated change in energy.

Brick motions that lower the energy are always accepted, and a small fraction of brick motions that raise the energy are accepted with the probability $P=\exp \left[-\Delta E / E_{o}\right]$, where $\Delta E$ is the energy associated with the brick motion and $E_{o}$ is a scaling factor that is analogous to $k T$ in simulations of physical annealing. This variant of Monte Carlo minimization is often referred to as 'simulated annealing', with the annealing temperature (T) dictating the probability of accepting an energy raising brick motion (Corana et al., 1987; Ingber, 1993). Complete details of the computational scheme are given in Lim et al. (2015). While displacements could be applied in a single step and the energy minimized, here the prescribed boundary conditions are applied incrementally and the energy is minimized for each (fictitious) time step. This has the advantage of capturing the evolution of damage as the applied displacements are increased.

Further, it can be used to dramatically speed up convergence, as follows: after several convergent load steps, the bricks are moved to new positions (prior to any Monte Carlo minimization), based on linear extrapolation of previous solutions to the new values of applied displacements. This significantly reduces the number of Monte Carlo iterations required to minimize the energy, by avoiding the need to migrate brick positions to their new equilibrium position using small Monte Carlo perturbations (Lim et al., 2015). This heuristic approach is motivated by linear elasticity; as will be illustrated, only a small portion of the entire sample (i.e. the region near the crack tip) experiences non-linear cohesive behavior, with the vast majority exhibiting linear elastic response. Hence, brick motions that are based on a linear extrapolation of previous solutions (imposed prior to the minimization step) are accurate for vast majority of the bricks.

Although direct search algorithms can circumvent several numerical stability issues resulting 
from potentially unreliable derivatives in gradient based schemes (Conn et al., 2009), they are by nature computationally expensive. Furthermore, this computational cost can increase significantly for systems with large numbers of degrees of freedom. However, direct search schemes can be highly amenable to parallelization (Lim et al., 2015). In the present parallelization scheme, the complete set of bricks is separated into sub-sets of non-adjacent bricks using a graph coloring algorithm (Gonzalez, 2007), which occurs just once during the initialization phase of the analysis. The individual groups (colors) of non-adjacent bricks are then sent to separate processor threads, and the calculation of energy changes associated with individual brick motions are performed in parallel while avoiding the simultaneous motion of adjacent bricks. This leads to a dramatic speedup over a corresponding sequential version; full details of the parallelization algorithm and performance relative to sequential computing are given in Lim et al. (2015).

For the results described here, around 200,000 bricks are used in a single simulation with 50 loading increments. At each load increment, the energy convergence was measured by automatically computing the variance and R-squared values from a statistical sample of energy values over the Monte Carlo cycle history and comparing to threshold tolerance parameters (Lim et al., 2015). These convergence parameters were set by trial and error such that the overall system energy did not change by more than a fraction of a percent upon switching to a more restrictive set of convergence parameters. For a typical set of convergence parameters (R-squared and variance threshold values of .02 and .002, respectively, over a sliding window of 3000 cycles), around 2030,000 Monte Carlo perturbations (each representing a complete set of brick trial motions) were required in the elastic regime, and around 60-70,000 Monte Carlo perturbations were required in the elastic/plastic and full fracture regime.

\subsection{Virtual tests}

Results are presented for two types of virtual tests: (i) a rectangular panel subjected to simple tension, and (ii) a square panel with a pre-defined macroscopic edge crack subjected to a combination of bending and tension. In the tension tests, the panel is loaded by moving the top row of bricks with a spatially uniform normal displacement. The average composite stress is then computed from the derivative of the global energy with respect to the macroscopic strain, defined as the applied displacement divided by the height of the specimen. The modulus is then computed from the second derivative of the global energy with respect to macroscopic strain. The reported 
strength corresponds to the peak stress obtained as the applied displacement is increased from zero to the point the panel ruptures.

For fracture simulations, a pre-crack is defined by zeroing the interface potential for all interfaces intersecting a straight line emanating from the left edge, as shown in Figure 1. The specimen is loaded by applying a rotation $\theta_{a p p}$ to the top and bottom faces of the panel. At the top of the panel (with $\mathrm{x}=0$ at the left edge of the specimen), this corresponds to:

$$
\underline{u}_{t}\left(x, \theta_{\text {app }}\right)=\left\{\operatorname{Sin}\left[\frac{\theta_{a p p}}{4}\right]^{2}\left(-x+\frac{H}{\theta_{a p p}}+H\right),-\frac{H}{2}+\operatorname{Sin}\left[\frac{\theta_{a p p}}{2}\right]\left(-x+\frac{H}{\theta_{a p p}}+H\right)\right\}
$$

with equal magnitude displacements at the bottom of the panel in the opposite direction. This loading condition corresponds to bending superposed with a small level of tension. (Note that the anisotropy of the material makes it difficult to identify a pure bending state a priori).

The macroscopic energy release rate is computed numerically, by running purely elastic simulations for various crack lengths, and calculating the macroscopic energy release rate as $G=$ $-\partial \Phi(a) / \partial a$, where $a$ is the crack length. (Purely elastic simulations are run by setting the cohesive yield stress to a large number that is not reached anywhere in the simulation). In the present simulations where yielding and rupture are allowed to occur, the size of the damage zone (where interface separations exceed $\Delta_{Y}$ ) is kept small relative to the size of the specimen, such that smallscale yielding conditions prevail. In these situations, the macroscopic driving force for crack growth is dictated by the elastic field surrounding the crack tip. The initiation fracture toughness is calculated by determining the value of the applied displacement needed to advance a pre-crack past a range of 1-5 bricks (i.e. rupturing the cohesive zones between multiple bricks), and computing the value of $G$ corresponding to this applied displacement. The small range of crack advance that defines fracture initiation represents the error bars in Figure 12A. As illustrated, this error was less than the size of the plot data point itself in most cases.

It is worth noting that the present approach of computing the macroscopic energy release rate (using purely elastic calculations involving different crack lengths) is somewhat unusual in the study of microstructural effects. An attractive alternative, applicable only to small-scale yielding, is to define a disk of material surrounding the crack and apply asymptotic displacements along the outer boundary that are defined in terms of applied crack tip stress intensity factors (or energy release rate) and the anisotropic elastic constants (Pardoen and Hutchinson, 2003; Zavattieri et al., 2008). This was not done in the present work because the asymptotic crack tip fields for the 
current material idealization have not yet been developed and are complicated by the presence of an internal length-scale not present in conventional continuum descriptions. Furthermore, the present approach is required in cases that do not invoke small-scale yielding, which is suggested by some fracture experiments (Launey et al., 2009) and is the focus of on-going simulations.

\section{Results and Discussion}

\subsection{Modulus and strength (without macroscopic pre-cracks)}

The uniaxial elastic modulus and macroscopic strength of a uniform panel of bricks is shown in Figure $4 \mathrm{~A}$ as a function of brick orientation relative to the loading direction. A uniform panel of $10^{5}$ bricks is loaded by displacing the bricks at one end of the specimen by a prescribed amount while holding the other edge fixed; in these and all results presented here, the brick overlap is taken to be $s=w / 2$. The results illustrate that the modulus and strength of the material loaded transverse to the brick direction ( $0^{\circ}$ orientation) is simply that expected from an elementary analysis of a two phase laminate loaded normal to the direction of the laminate. That is, the modulus is simply the brick height $(h)$ multiplied by the cohesive stiffness $\left(k_{n}\right)$, while the strength is simply the peak strength in the cohesive law. As the orientation of the applied stress is brought in coincidence with the long brick direction, the effective modulus and strength increase due to shear transfer to the bricks; in the limit of $90^{\circ}$ loading (parallel to the brick direction), one obtains the enhanced properties predicted by micromechanical models presented elsewhere for rigid bricks (Begley et al., 2012; Bertoldi et al., 2008; Ji and Gao, 2004). As shown in Figure 4, the enhancement in both modulus and strength is a strong function of brick aspect ratio $(w / h)$, as previously predicted. We note that the transition between these two limits involves brick rotations, which have not been included in previous models. Also note that there is a small error (3\% at most) in the zero degree modulus calculations when compared to the theoretical results. It was determined that this error was due to a non-zero Monte Carlo temperature used as a simulation parameter. The error was reduced to less than half of a percent with a lower Monte Carlo temperature, at the expense of added computational time. 

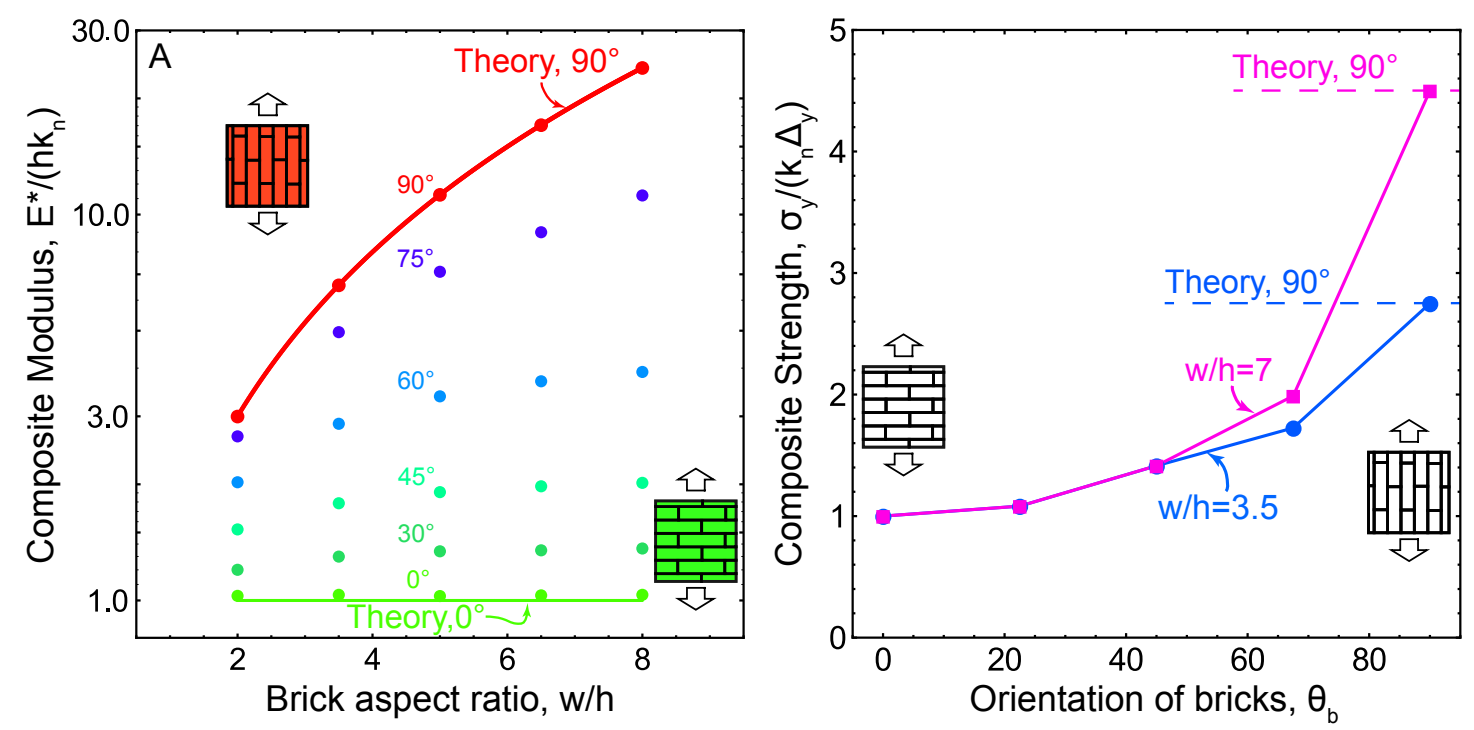

Figure 4: (a) Macroscopic composite modulus calculated from total system energy in tensile specimen, and (b) macroscopic strength computed from maximum load in tension test.

\subsection{Crack tip behavior: stresses and damage}

Figure 5 shows the stresses ahead of a macroscopic crack acting normal to the crack plane $\left(\sigma_{22}\right)$, for a purely elastic solid in which interface yielding and rupture is prevented. The total number of bricks is $\sim 2.3 \times 10^{5}$, with the brick height $(h)$ defined as one unit and all other length-scales defined relative to that size. In Figure 5, note the scale bar indicating the length scale of two hundred twenty bricks in the $90^{\circ}$ orientation: in the $0^{\circ}$ orientation, this same scale bar spans roughly thirty bricks (along the $w$ brick dimension). The stresses shown in Figure 5 (and all subsequent figures) represent the average volumetric brick stresses due to surrounding interfacial tractions, computed via the surface integral:

$$
\bar{\sigma}_{i j}=\sum_{k=1}^{n} \frac{1}{w h} \int_{S} x_{j}(s) T_{i}(s) d s
$$

where $x_{j}(s)$ are the components of the position vector, $T_{i}(s)$ are the components of the traction vector, and $n$ is the total number of interfaces surrounding the brick (which for a regular array of bricks is six for all interior bricks). The level of applied displacements in Figure 5 corresponds to those that would cause crack advance for a set of cohesive rupture properties considered later, even though yielding is not permitted. The stress fields are analogous to those obtained for conventional anisotropic elastic materials (assuming a homogeneous distribution of properties). The size of the region ahead of the crack that sees large stresses is a strong function of orientation, due to load 
transfer between bricks.

Note that there are small stress jumps from one brick to the next, as evidenced by the somewhat roughened appearance of the contours. This is due to the fact that a very minute proportion of energy raising brick motions are actually allowed in the final displacement configuration, which results from a non-zero annealing temperature. It has been verified via simulation that as the global temperature is reduced, the contours appear much smoother, as less energy raising motions are allowed by the brick energy acceptance criteria. These variations in brick stresses have a negligible impact on the resulting fracture behavior; hence, eliminating them increases computational cost without much benefit.

Figures 6 and 7 show stress contours at the crack tip ( $\sigma_{22}$ and $\sigma_{12}$ respectively), for the exact same applied displacements as shown in Figure 5, only now with a full elastic-plastic-rupture cohesive description. In these figures, $\Delta_{Y} / h=0.01$ and $\Delta_{R} / h=31.42$, and the applied displacements correspond to those just at the threshold of crack advance for this set of properties. The impact of cohesive properties is discussed later. By comparison with the elastic results in Figure 5, one can see the impact of interface yielding on altering the distribution of stresses ahead of the crack tip. Specifically, one observes the formation of strong discontinuities in the brick stresses at angles that correspond to lines running through the mid-point of brick sides to the mid-point of brick ends. To provide additional perspective regarding the extent of damage, Figure 8 shows contours where the bricks are colored by the number of interfaces on the brick perimeter that have exceeded their yield strain.

Figure 9 shows a log-log plot of the average brick stresses along the horizontal plane ahead of the crack tip, for cases with two different brick aspect ratios. Two features of the stress distribution are immediately apparent: the stress fields exhibit a plateau in the damage region, and they exhibit distributions outside the damage region that are close to the classic $1 / \sqrt{r}$ distributions from elasticity theory. This behavior is consistent with the notion of small-scale yielding being controlled by a dominant elastic field. Note that for the smaller aspect ratio bricks $(w / h=3.5)$ and $90^{\circ}$ orientation, there is an intermediate zone (between the 'plastic zone' and 'elastic zone') where the stresses are less singular than $1 / \sqrt{r}$ : the source of this behavior has yet to be elucidated. The scaling of the damage region (effectively a plastic zone size) with system parameters is discussed in the next section. 

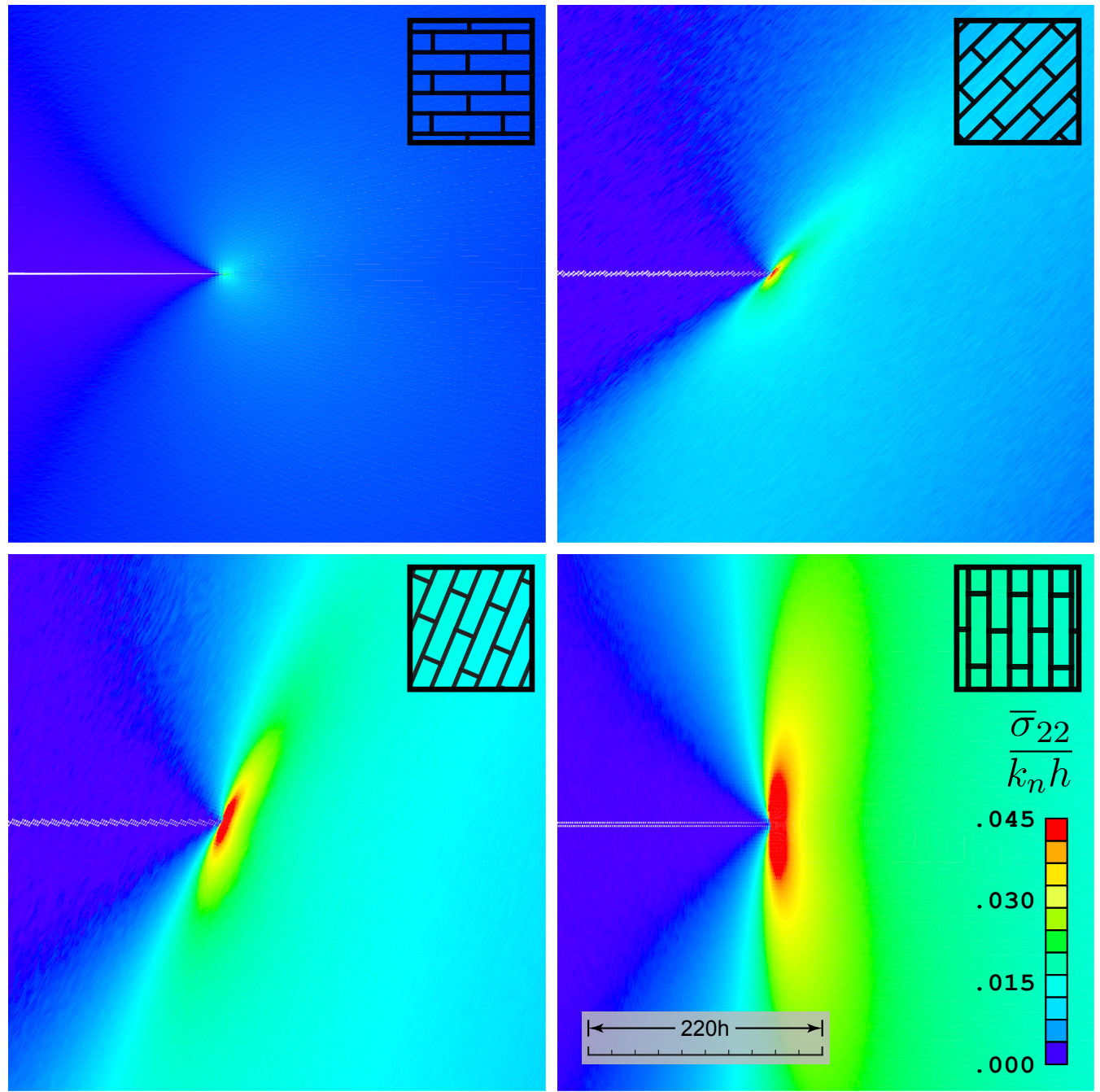

Figure 5: Direct stress $\left(\sigma_{22}\right)$ contours near crack tip for elastic specimen with $\theta_{b}=0^{\circ}, 45^{\circ}, 67.5^{\circ}$, and $90^{\circ}$, with $w / h=7, \Delta_{Y} / h=\infty, W / H=1, a_{0} / W=0.33, N_{b} \approx 225000$, shown at $5 \mathrm{x}$ magnification. Contour range is set such that the minimum and maximum color bands represent the same absolute (unscaled) stress range as in Figure 6. Frames are taken at the same loading value as fracture simulations in Figures 6,7 , and 8 , e.g., $G \approx G_{c}$ even though rupture is not allowed. Rendered using OpenGL 4 on a single Nvidia GTX580 graphics processing unit with full-screen anti-aliasing. 

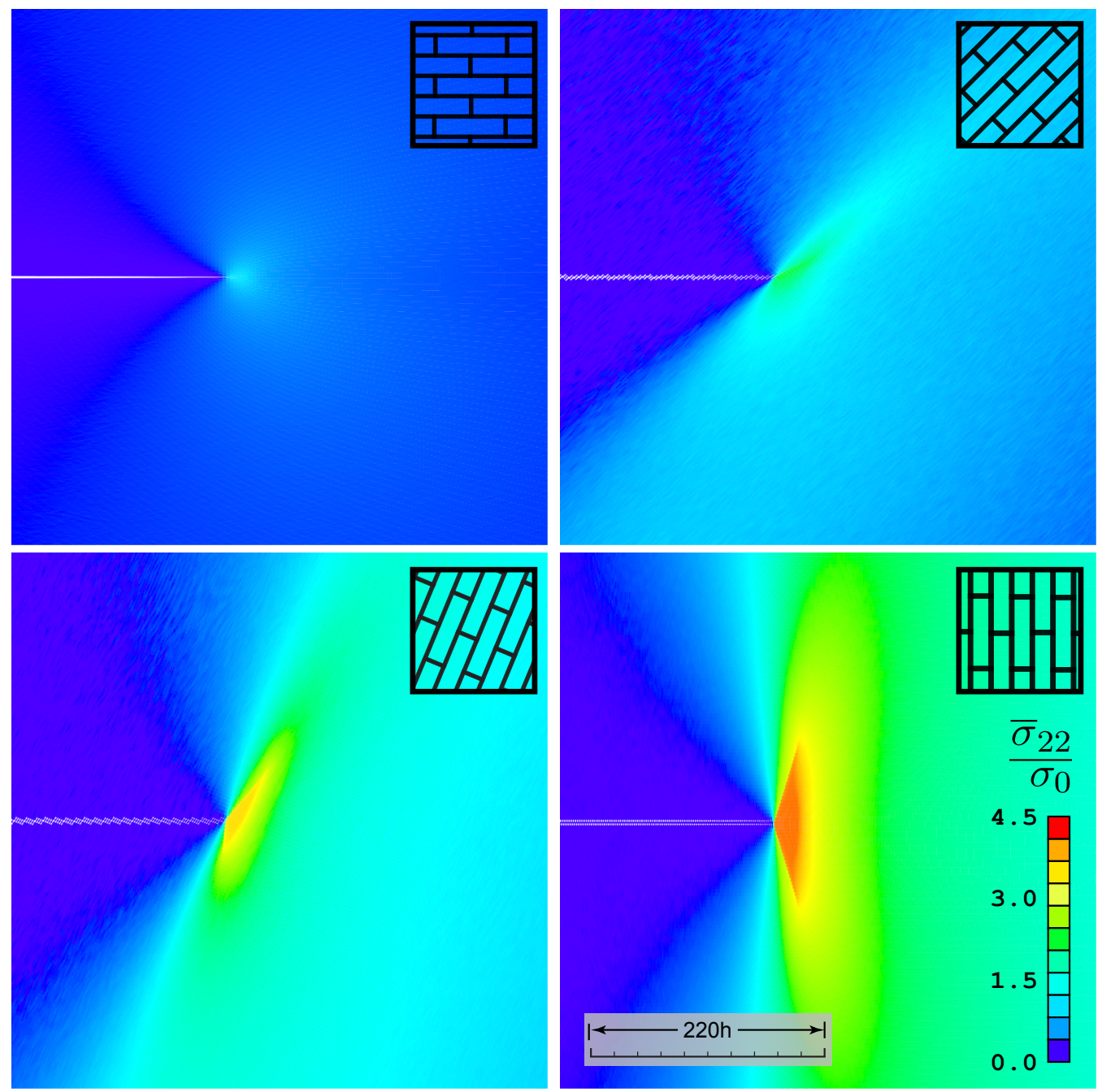

Figure 6: Direct stress $\left(\sigma_{22}\right)$ contours near crack tip for a full fracture specimen with $\theta_{b}=0^{\circ}, 45^{\circ}, 67.5^{\circ}$, and $90^{\circ}$, with $w / h=7, \Delta_{Y} / h=0.01, \Delta_{R} / \Delta_{Y}=31.42, W / H=1, a_{0} / W=0.33, N_{b} \approx 225000$, shown at 5x magnification. Brick stresses scaled by cohesive interface strength, $\sigma_{0}=k_{n} \Delta_{Y}$. Frames are taken immediately prior to fracture initiation, e.g., $G \approx G_{c}$. 

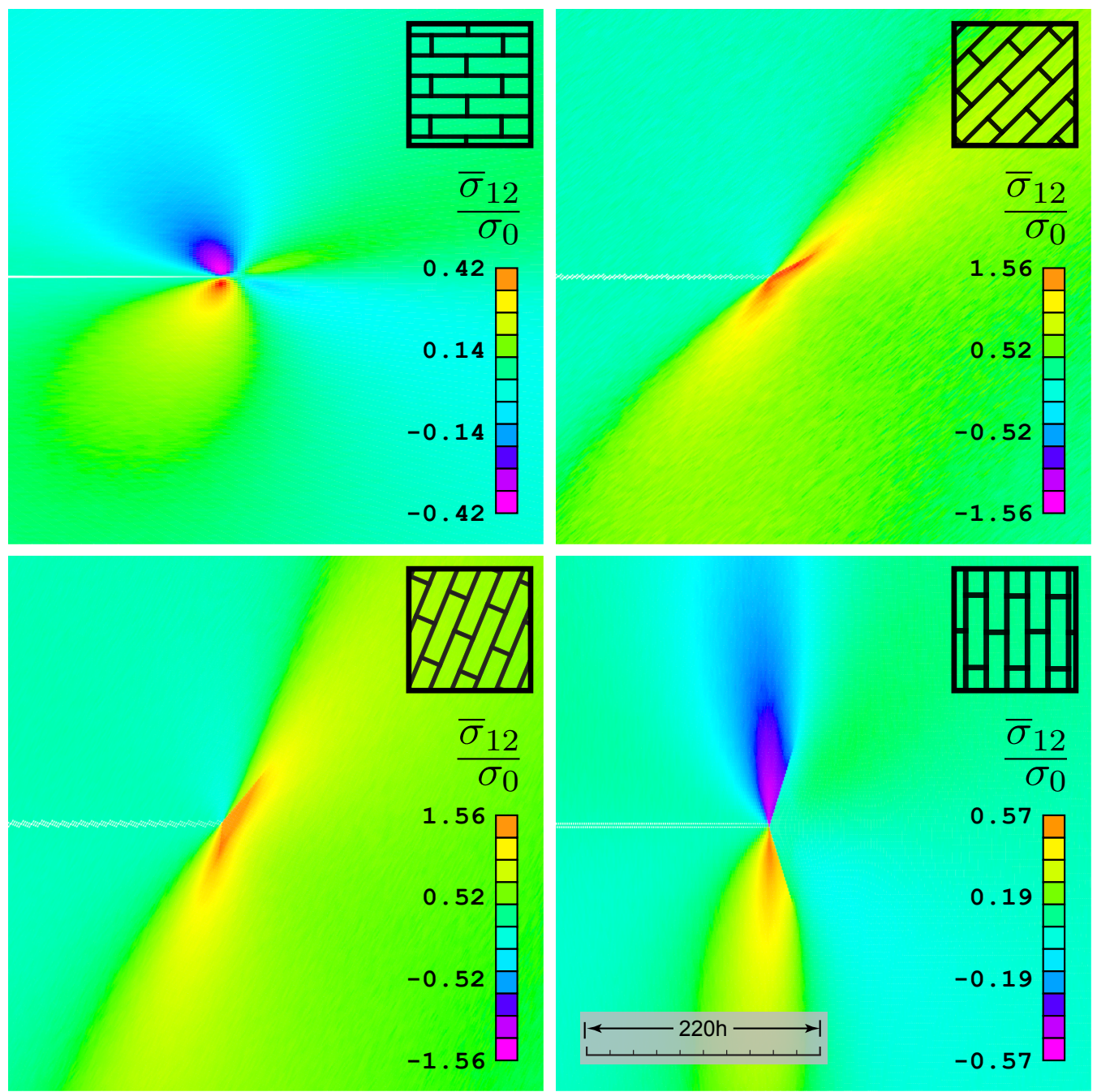

Figure 7: Shear stress $\left(\sigma_{12}\right)$ contours near crack tip for a full fracture specimen with $\theta_{b}=0^{\circ}, 45^{\circ}, 67.5^{\circ}$, and $90^{\circ}$, with $w / h=7, \Delta_{Y} / h=0.01, \Delta_{R} / \Delta_{Y}=31.42, W / H=1, a_{0} / W=0.33, N_{b} \approx 225000$, shown at 5x magnification. Brick stresses scaled by cohesive interface strength, $\sigma_{0}=k_{n} \Delta_{Y} . G \approx G_{c}$. 

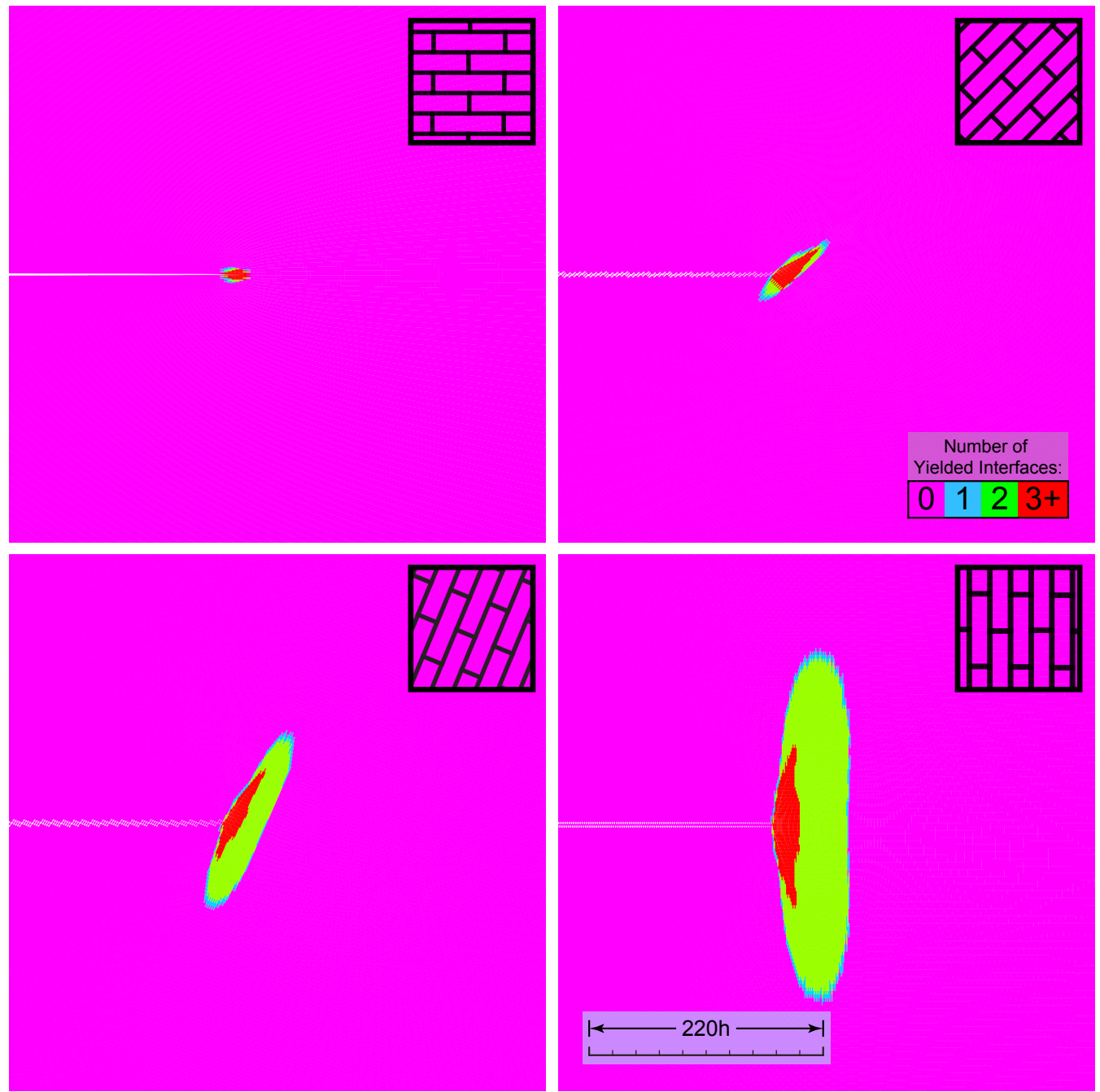

Figure 8: Damage distribution near crack tip for full fracture specimen with $\theta_{b}=0^{\circ}, 45^{\circ}, 67.5^{\circ}$, and $90^{\circ}$, and $w / h=7, \Delta_{Y} / h=0.01, \Delta_{R} / \Delta_{Y}=31.42, W / H=1, a_{0} / W=0.33, N_{b} \approx 225000$, shown at 5x magnification. Colors represent number of adjacent yielded interfaces, e.g., $|\underline{\Delta}|_{i, a v e} \geq \Delta_{Y}$. $G \approx G_{c}$. 

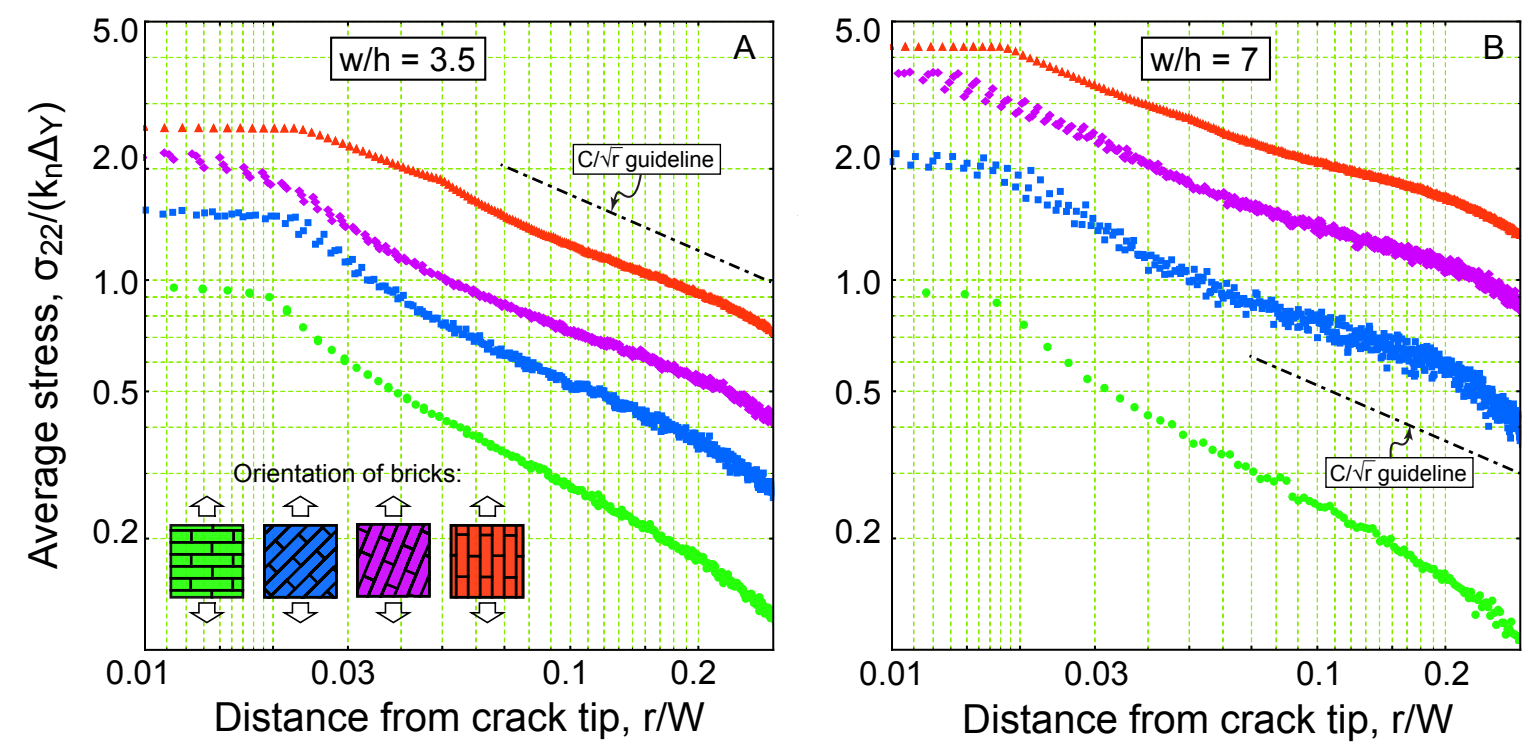

Figure 9: Direct stress $\sigma_{22}$ distributions for (a) $w / h=3.5$ and (b) $w / h=7$, for various orientations $\theta_{b}=0^{\circ}, 45^{\circ}, 67.5^{\circ}$, and $90^{\circ}$, and $\Delta_{Y} / h=0.01, \Delta_{R} / \Delta_{Y}=31.42, W / H=1, a_{0} / W=0.33, N_{b} \approx 225000 . G \approx G_{c}$.

\subsection{Toughness as a function of microstructure}

The energy release rates $(G)$ computed for purely elastic specimens subjected to the loading given in Figure 1 are shown in Figure 10A as a function of orientation and crack length. The results in the main figure are normalized by the square of the applied displacement parameter; conversely, the inset illustrates that the energy release varies with the square of the applied displacement parameter $\theta_{a p p}$ when the brick size and interface stiffness are used to normalize $G$. Figure 10B shows the macroscopic initiation fracture toughness that is inferred from simulations that allow for interface rupture, using the critical value of applied displacements that leads to complete rupture of at least five bricks and the elastic results shown in Figure 10A. Error bars in Figure 10B represent the error due to size of the discrete loading steps (increments in applied displacement) and discrete advances in crack length, and are smaller than the plotting points in this case. The fact that a consistent toughness is obtained as a function of crack length validates the interpretation that the inferred toughness is a material property and is independent of specimen geometry. Indeed, additional calculations not shown here for pure remote tension of an edge-cracked panel yield the same numbers as shown in Figure 10B. This specimen-independence is a consequence of the fact that small-scale yielding conditions are enforced, by choosing panel dimensions that are much larger 
than damage zones near the macroscopically defined pre-crack.

Figure 11A shows the inferred toughness for the $90^{\circ}$ orientation as a function of the mortar rupture strain (recall that $\epsilon_{R}=t \Delta_{R}$, for fixed yield strain $\Delta_{Y}$ ). The corresponding size of the damage zone is shown in Figure 11B, using an effective plastic zone radius $r_{p}$. It can be shown that the size of the damage zone in bricks $\left(r_{p} / h\right)$ scales directly with the ductility of the interface, measured by ratio of the cohesive rupture separation $\left(\Delta_{R}\right)$ to the critical separation $\left(\Delta_{Y}\right)$ :

$$
\frac{r_{p}}{h}=\frac{\Delta_{R}}{\Delta_{Y}} f\left(\frac{w}{h}, \theta_{b}\right)
$$

This linear scaling with $\Delta_{R} / \Delta_{Y}$ is analogous to that obtained for plastic zone sizes in isotropic elastic materials under the assumption that yielding occurs where the elastic fields exceed the yield stress of the material. In Figure 11B, note that at large values of interface ductility and large aspect ratios, the scaling of the plastic zone appears to change slightly, growing faster than the expected linear relationship.

The results in Figure 11 illustrate the consequences of the number of bricks in the damage zone $\left(N_{p}\right)$, as controlled by $\Delta_{R} / \Delta_{Y}$ : as the interface ductility is increased, the behavior within the plastic zone is resolved over a larger number of bricks, and the toughness asymptotes to a constant value for $\Delta_{R} / \Delta_{Y} \approx 20-30$, which corresponds to plastic zones that are approximately one hundred bricks wide in linear dimension. It should be noted that the unscaled value of the composite initiation toughness $\left(G_{c}\right)$ increases linearly with interface ductility, since the intrinsic toughness $\Gamma_{i}$ of the interfaces also increases linearly with the interface ductility.

The key feature of Figure 11A is that the relative increase of the macroscopic toughness to that of the interfaces becomes constant in the limit of large interface ductility. This is a consequence of the fact that the behavior in the fracture process zone is controlled by a transition region between the crack tip and the far field elastic results, which is fully resolved in the sense that the number of bricks in this zone is immaterial. This can be viewed as the 'small microstructure' limit, wherein the bricks are small enough to not individually influence the fracture behavior. In this limit, the initiation fracture toughness is controlled by behaviors that span hundreds of bricks. Put another way, at low values of interface ductility, the number of bricks in the region controlling the fracture process is rather limited and one observes fracture prior to the development of widespread 'plasticity'.

Finally, Figure 12 illustrates the initiation toughness of the material for cracks with various 

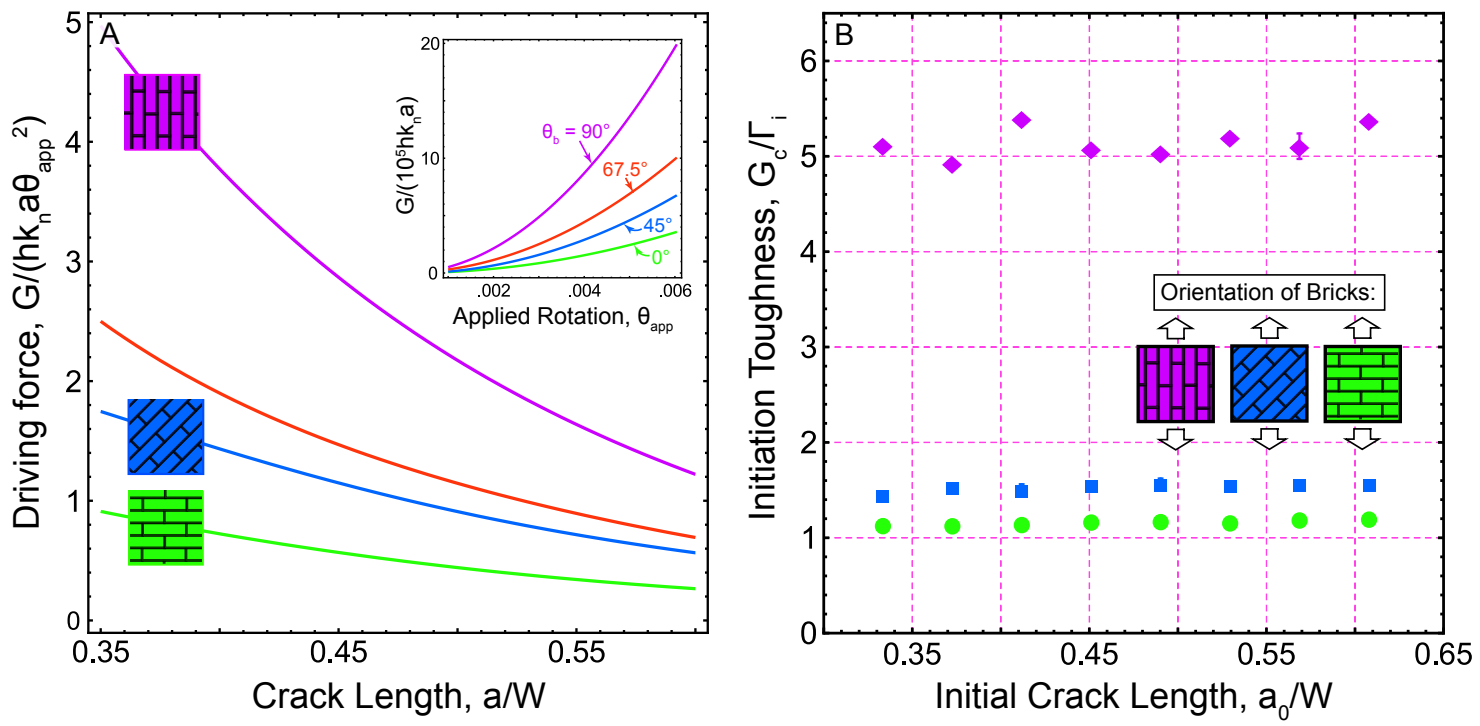

Figure 10: (a) Macroscopic energy release rate (G), computed via multiple elastic simulations at different crack lengths and (b) toughness values from different initial flaw sizes. $\Delta_{Y} / h=\infty$ (left), and $\Delta_{Y} / h=0.01, \Delta_{R} / \Delta_{Y}=31.42$ (right). For both cases, $W / H=1, a_{0} / W=0.33, N_{b} \approx 225000, w / h=3.5$, and $\theta_{a p p}=.00604$.

orientation angles relative to that of the bricks. These results reflect the 'limit' toughness, i.e. that obtained for a rupture strain large enough to ensure that the damage zone encompasses enough bricks to resolve the behavior in the plastic zone. As one might expect, the toughness of the material for cracks that are aligned with the bricks is identical to that of just the interface; in this orientation, the microstructure bears no benefit. However, as the orientation mismatch between the crack and brick microstructure increases, toughness increases dramatically due to crack bridging. The initiation toughness for cracks running transverse to the microstructural direction is 5-10 times higher than the intrinsic toughness $\Gamma_{i}$, or work to failure of the mortar material. Note that for shorter bricks the benefit of alignment is only pronounced only at crack orientations very close to perpendicular to the brick direction. While the range of beneficial orientations increases with brick aspect ratio, there is nevertheless a strong dependence on brick orientation as seen in Figure 12A.

The simulations make no a priori assumption about the direction of crack growth, and one observes several transitions in the angles between the microstructure, the orientation of the pre-crack, and the crack growth direction. As illustrated in Figure 12B, four different failure mechanisms can be identified from the simulation results: splitting, angle splitting, stairway, and columnar. These agree with previously documented failure mechanisms (Rabiei et al., 2010). For small orientation 

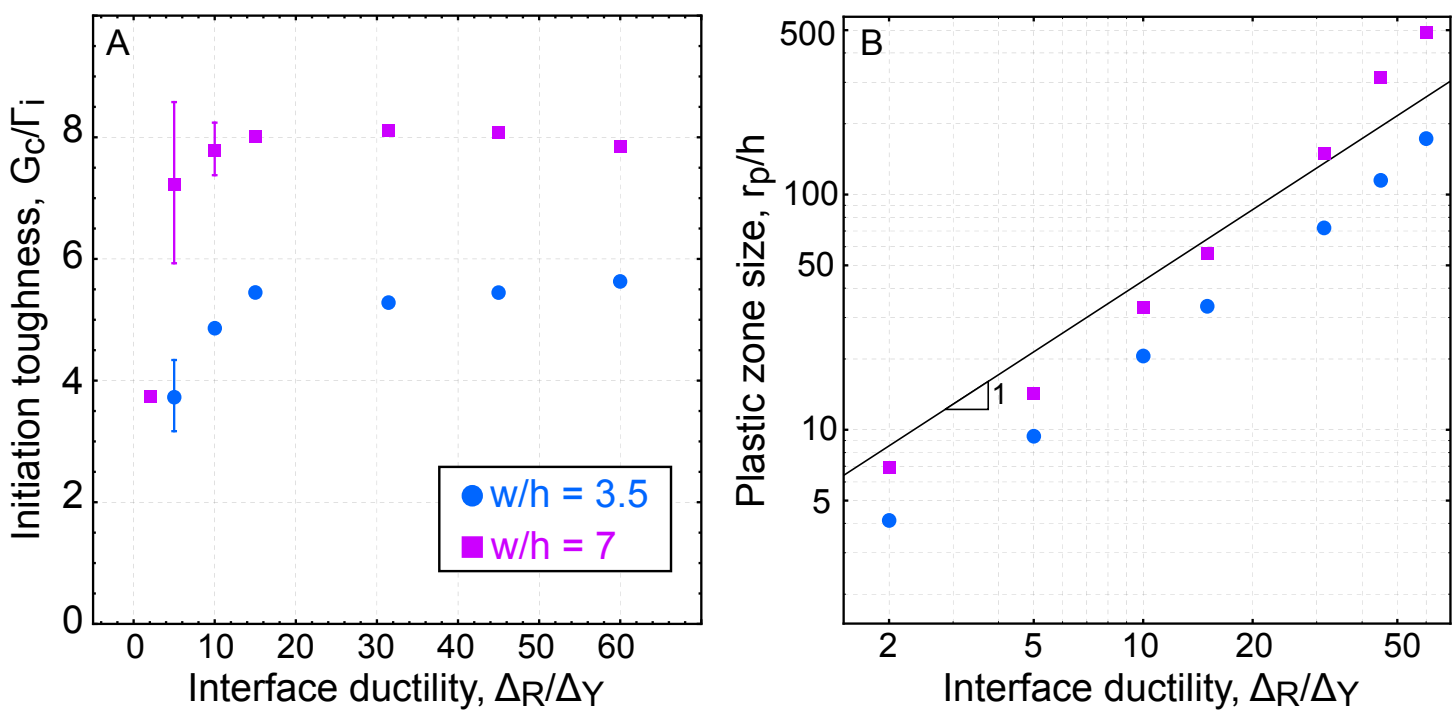

Figure 11: (a) Effect of interface ductility on macroscopic initiation toughness and (b) effective plastic zone radius at fracture initiation, where $\frac{r_{p}}{h}=\sqrt{\left(\frac{w}{h}\right) N_{p}}$, and $N_{p}$ is the number of bricks in the plastic zone. For both cases, $\theta_{b}=90^{\circ}$.

differences between microstructure and pre-crack, the cracks advance along the long faces of the bricks, with essentially no benefit from the microstructure. For larger differences, the crack first turns and advances in a staircase pattern, with the macroscopic direction of crack advance essentially dictated by the angle formed by a line running between the midpoints of the brick sides. For purely perpendicular mismatch between the crack and brick direction, one observes crack paths that are essentially straight, such that bridging ahead of the dominant crack produces significant enhancements in toughness.

While the toughness of synthetic nacres has not yet been measured as a function of pre-crack orientation, measurements of the R-curves for crack growth in the direction perpendicular to the bricks enable a comparison of theory and experiment. In the $\mathrm{Al}_{2} \mathrm{O}_{3} / \mathrm{PMMA}$ composites in (Munch et al., 2008), the initiation toughness is about $700 \mathrm{~J} / \mathrm{m}^{2}$ (This value is obtained by converting the published values of $K_{c}$ to $G_{c}$ using the measured composite modulus). While the cohesive properties of the mortar were not measured, to a first approximation we can assume that the intrinsic toughness of the mortar materials is comparable to that of bulk PMMA, taken here to be $300 \mathrm{~J} / \mathrm{m}^{2}$ (Choi and Salem, 1993). This implies that the experimental measurement of $G_{c} / \Gamma_{i}$ is in the neighborhood of $2-3$. This is significantly smaller than that shown in Figure $12 \mathrm{~A}$, i.e. $G_{c} / \Gamma_{i} \sim 5-10$, however, 
in the present simulations, all bricks are perfectly aligned and have the exact same shape and size. Real materials, either biological or synthetic, will exhibit a stochastic distribution of brick sizes and overlap, which can have a profound impact on the predicted properties (Zhang et al., 2010a; Lei et al., 2012). We recently have begun to conduct simulations with Gaussian distributions of brick sizes throughout the microstructure; this leads to widely-spaced regions of aligned vertical interfaces in the short direction of the bricks, which appear to act as defects. Our preliminary simulations to be published indicate that even small variations in brick sizes and mortar properties can lead to significant reductions in strength and toughness, on the order of $50 \%$ in some instances. This suggests that local microstructural features have a large impact on observed toughness. That is, small regions where brick ends are aligned, rather than staggered, can have a large impact on predicted toughness.

It is interesting to note, however, that the measured R-curve behaviors for synthetic nacrelike composites show significant increase in toughness beyond initiation. It is possible that the R-curve behavior will be less sensitive to local defects, due to the fact that larger material volumes are sampled as the crack extends. Additional simulations of local microstructural defects and Rcurve behavior are needed to fully understand these effects; this would require that the simulation approach be adapted to account for inelastic unloading of damaged interfaces.

\section{Concluding remarks}

The present work presents a computational framework to predict the impact of brick shape and alignment on the relationship between macroscopic toughness and inelastic behaviors between bricks, i.e. the work-to-failure of the interface between bricks. The method is highly flexible with regards to handling bricks of different shapes and distributions, and creates interesting opportunities to explore the effect of material hierarchy (Zhang et al., 2010b). For example, one could use the current approach to study bricks within bricks, i.e. smaller patterned features within bricks, with different cohesive laws inside and between macro- and micro-scale bricks. The efficiency and robustness of the present approach implies considerable advantages over finite element models of such studies of material hierarchy. Implementation of the model and direct search minimization algorithm within a GPU platform results in an efficient framework that can be used to conduct broad parameter studies to guide future materials development. 


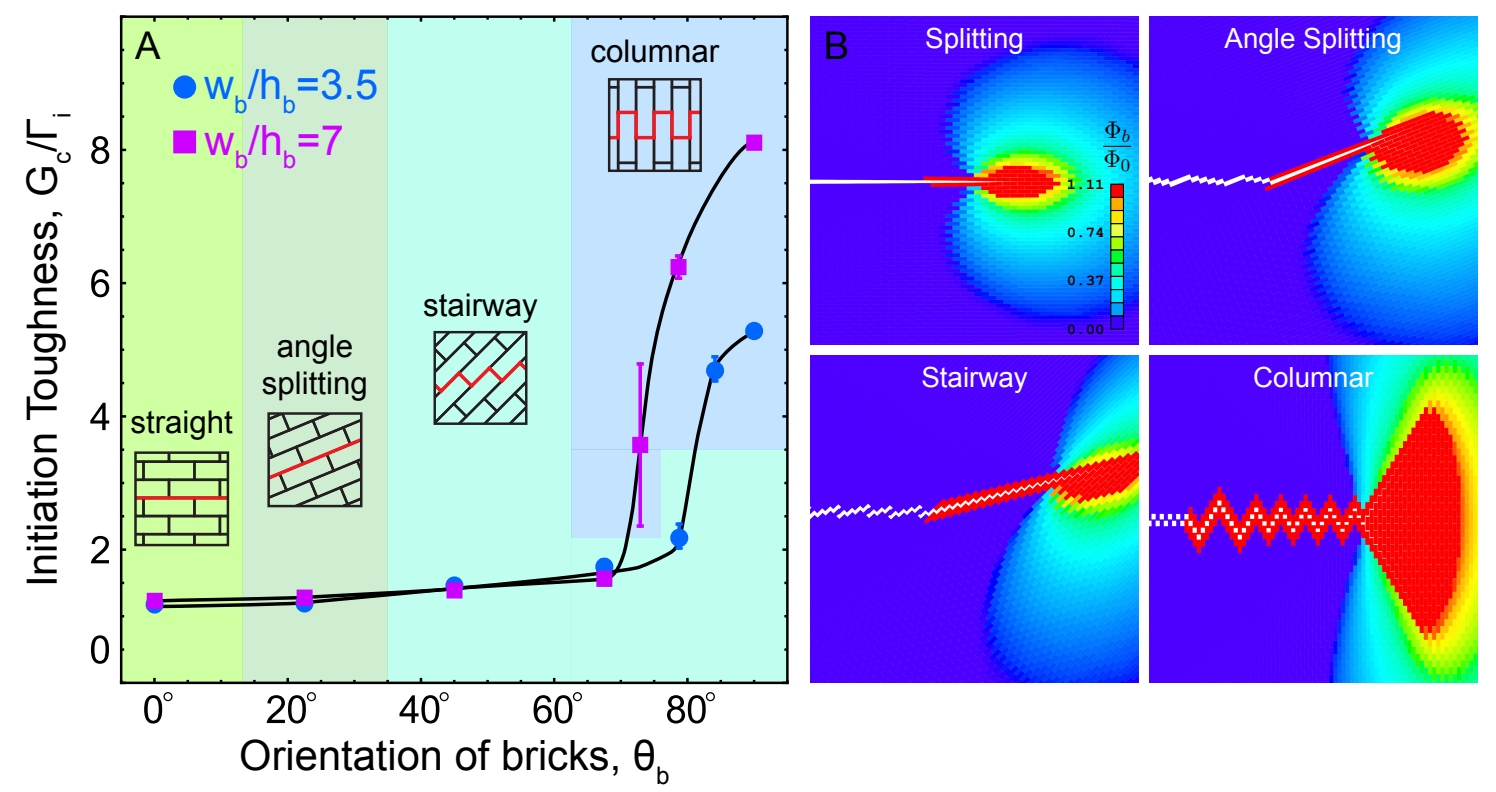

Figure 12: (a) Macroscopic fracture initiation toughness for all brick orientations and aspect ratios and (b) simulated failure mechanisms, with $\Delta_{Y} / h=0.01, \Delta_{R} / \Delta_{Y}=31.42, W / H=1, a_{0} / W=0.33, N_{b} \approx 225000$. Colors contours in (b) represent the total interface energy surrounding a brick $\left(\Phi_{b}\right)$ scaled by the maximum allowable elastic energy of all those interfaces $\Phi_{0}$, where $\Phi_{0}=(w+h) k_{n} \Delta_{Y}^{2}$.

The framework is currently limited to microstructures for which the bricks can be treated as rigid, which limits its application to synthetic materials whose modulus mismatch between mortar and bricks is large and whose volume fraction of mortar (as manifest as mortar thickness) is about 5-10\%. Nevertheless, the simulations demonstrate that one obtains anistropic elastic fields that control the energy dissipated in the fracture process zone, and that the corresponding toughness enhancement is a strong function of orientation. For such materials, the simulations show strong agreement between the predicted gain in macroscopic toughness and that observed from experiments, at least for the $90^{\circ}$ loading orientation. Future work is needed to incorporate different unloading behaviors to predict R-curve behavior, as well as the impact of brick deformation (including failure). 


\section{Acknowledgements:}

This research was financially supported through grant DMR-1105672 from the National Science foundation and grant W911NF-09-0001 through the Institute for Collaborative Biotechnologies from the U.S. Army Research office. Additional computational resources were provided by the Center for Scientific Computing at the CNSI and MRL: an NSF MRSEC (DMR-1121053) and NSF CNS-0960316. The content and information presented does not reflect the position or policy of the U.S. government, and no official endorsement should be inferred or implied.

\section{References:}

Allen, M. P., Tildesley, D. J., et al., 1987. Computer simulation of liquids.

An, B., Zhao, X., Arola, D., Zhang, D., 2014. Fracture analysis for biological materials with an expanded cohesive zone model. Journal of biomechanics.

Barthelat, F., 2007. Biomimetics for next generation materials. Philosophical Transactions of the Royal Society A: Mathematical, Physical and Engineering Sciences 365 (1861), 2907-2919.

Barthelat, F., Espinosa, H., 2007. An experimental investigation of deformation and fracture of nacre-mother of pearl. Experimental mechanics 47 (3), 311-324.

Barthelat, F., Rabiei, R., 2011. Toughness amplification in natural composites. Journal of the Mechanics and Physics of Solids 59 (4), 829-840.

Barthelat, F., Tang, H., Zavattieri, P., Li, C.-M., Espinosa, H., 2007. On the mechanics of motherof-pearl: a key feature in the material hierarchical structure. Journal of the Mechanics and Physics of Solids 55 (2), 306-337.

Begley, M. R., Philips, N. R., Compton, B. G., Wilbrink, D. V., Ritchie, R. O., Utz, M., 2012. Micromechanical models to guide the development of synthetic brick and mortarcomposites. Journal of the Mechanics and Physics of Solids 60 (8), 1545-1560.

Bekah, S., Rabiei, R., Barthelat, F., 2012. The micromechanics of biological and biomimetic staggered composites. Journal of Bionic Engineering 9 (4), 446-456.

Bertoldi, K., Bigoni, D., Drugan, W., 2008. Nacre: an orthotropic and bimodular elastic material. Composites Science and Technology 68 (6), 1363-1375. 
Binder, K., Heermann, D., 2010. Monte Carlo simulation in statistical physics: an introduction. Springer.

Bouville, F., Maire, E., Meille, S., Van de Moortèle, B., Stevenson, A. J., Deville, S., 2014. Strong, tough and stiff bioinspired ceramics from brittle constituents. Nature materials 13 (5), 508-514.

Choi, S., Salem, J., 1993. Fracture toughness of pmma as measured with indentation cracks. Journal of Materials research 8 (12), 3210-3217.

Conn, A. R., Scheinberg, K., Vicente, L. N., 2009. Introduction to derivative-free optimization. Vol. 8. Siam.

Corana, A., Marchesi, M., Martini, C., Ridella, S., 1987. Minimizing multimodal functions of continuous variables with the simulated annealing algorithm corrigenda for this article is available here. ACM Transactions on Mathematical Software (TOMS) 13 (3), 262-280.

Currey, J., 1977. Mechanical properties of mother of pearl in tension. Proceedings of the Royal Society of London. Series B. Biological Sciences 196 (1125), 443-463.

Deville, S., Saiz, E., Nalla, R. K., Tomsia, A. P., 2006. Freezing as a path to build complex composites. Science 311 (5760), 515-518.

Evans, A., Suo, Z., Wang, R., Aksay, I., He, M., Hutchinson, J., 2001. Model for the robust mechanical behavior of nacre. Journal of Materials Research 16 (09), 2475-2484.

Finnemore, A., Cunha, P., Shean, T., Vignolini, S., Guldin, S., Oyen, M., Steiner, U., 2012. Biomimetic layer-by-layer assembly of artificial nacre. Nature communications 3, 966.

Flores-Johnson, E., Shen, L., Guiamatsia, I., Nguyen, G. D., 2014. Numerical investigation of the impact behaviour of bioinspired nacre-like aluminium composite plates. Composites Science and Technology 96, 13-22.

Genet, M., Couégnat, G., Tomsia, A. P., Ritchie, R. O., 2014. Scaling strength distributions in quasi-brittle materials from micro-to macro-scales: A computational approach to modeling nature-inspired structural ceramics. Journal of the Mechanics and Physics of Solids 68, 93-106.

Gonzalez, T. F., 2007. Handbook of approximation algorithms and metaheuristics. CRC Press. 
Ingber, L., 1993. Simulated annealing: Practice versus theory. Mathematical and computer modelling 18 (11), 29-57.

Jackson, A., Vincent, J., Turner, R., 1988. The mechanical design of nacre. Proceedings of the Royal society of London. Series B. Biological sciences 234 (1277), 415-440.

Jackson, A., Vincent, J., Turner, R., 1990. Comparison of nacre with other ceramic composites. Journal of Materials Science 25 (7), 3173-3178.

Jäger, I., Fratzl, P., 2000. Mineralized collagen fibrils: a mechanical model with a staggered arrangement of mineral particles. Biophysical Journal 79 (4), 1737-1746.

Ji, B., Gao, H., 2004. Mechanical properties of nanostructure of biological materials. Journal of the Mechanics and Physics of Solids 52 (9), 1963-1990.

Kamat, S., Su, X., Ballarini, R., Heuer, A., 2000. Structural basis for the fracture toughness of the shell of the conch strombus gigas. Nature 405 (6790), 1036-1040.

Katti, D. R., Pradhan, S. M., Katti, K. S., 2004. Modeling the organic-inorganic interfacial nanoasperities in a model bio-nanocomposite, nacre. Reviews on Advanced Materials Science $6(2), 162-168$.

Kotha, S., Li, Y., Guzelsu, N., 2001. Micromechanical model of nacre tested in tension. Journal of materials science $36(8)$.

Launey, M. E., Munch, E., Alsem, D., Barth, H., Saiz, E., Tomsia, A. P., Ritchie, R. O., 2009. Designing highly toughened hybrid composites through nature-inspired hierarchical complexity. Acta Materialia 57 (10), 2919-2932.

Lei, H., Zhang, Z., Liu, B., 2012. Effect of fiber arrangement on mechanical properties of short fiber reinforced composites. Composites Science and Technology 72 (4), 506-514.

Lim, R. K., Pro, J. W., Begley, M. R., Petzold, L. R., Utz, M., 2015. High-performance simulation of fracture in idealized brick and mortar composites using adaptive monte carlo minimization on the gpu. Submitted to the International Journal of High Performance Computing. 
McMeeking, R. M., 1977. Finite deformation analysis of crack-tip opening in elastic-plastic materials and implications for fracture. Journal of the Mechanics and Physics of Solids 25 (5), 357-381.

Menig, R., Meyers, M., Meyers, M., Vecchio, K., 2001. Quasi-static and dynamic mechanical response of strombus gigas (conch) shells. Materials Science and Engineering: A 297 (1), 203-211.

Meyers, M. A., Chen, P.-Y., Lin, A. Y.-M., Seki, Y., 2008. Biological materials: structure and mechanical properties. Progress in Materials Science 53 (1), 1-206.

Munch, E., Launey, M. E., Alsem, D. H., Saiz, E., Tomsia, A. P., Ritchie, R. O., 2008. Tough, bio-inspired hybrid materials. Science 322 (5907), 1516-1520.

Nguyen, T., Govindjee, S., 2006. Numerical study of geometric constraint and cohesive parameters in steady-state viscoelastic crack growth. International journal of fracture 141 (1-2), 255-268.

Okumura, K., De Gennes, P.-G., 2001. Why is nacre strong? elastic theory and fracture mechanics for biocomposites with stratified structures. The European Physical Journal E 4 (1), 121-127.

Pardoen, T., Hutchinson, J., 2003. Micromechanics-based model for trends in toughness of ductile metals. Acta Materialia 51 (1), 133-148.

Pro, J. W., Lim, R. K., Begley, M. R., Petzold, L. R., Utz, M., 2015. Fracture simulations of heterogeneous brick and mortar microstructures. in preparation. In Preparation.

Rabiei, R., Bekah, S., Barthelat, F., 2010. Failure mode transition in nacre and bone-like materials. Acta biomaterialia 6 (10), 4081-4089.

Song, F., Soh, A., Bai, Y., 2003. Structural and mechanical properties of the organic matrix layers of nacre. Biomaterials 24 (20), 3623-3631.

Tang, H., Barthelat, F., Espinosa, H., 2007. An elasto-viscoplastic interface model for investigating the constitutive behavior of nacre. Journal of the Mechanics and Physics of Solids 55 (7), 14101438.

Vincent, J. F., 2003. Biomimetic modelling. Philosophical Transactions of the Royal Society of London. Series B: Biological Sciences 358 (1437), 1597-1603. 
Wang, R., Suo, Z., Evans, A., Yao, N., Aksay, I., 2001. Deformation mechanisms in nacre. Journal of Materials Research 16 (09), 2485-2493.

Wegst, U., Ashby, M., 2004. The mechanical efficiency of natural materials. Philosophical Magazine $84(21), 2167-2186$.

Wilbrink, D. V., Utz, M., Ritchie, R. O., Begley, M. R., 2010. Scaling of strength and ductility in bioinspired brick and mortar composites. Applied Physics Letters 97 (19), 193701.

Zavattieri, P. D., Hector Jr, L. G., Bower, A. F., 2008. Cohesive zone simulations of crack growth along a rough interface between two elastic-plastic solids. Engineering Fracture Mechanics 75 (15), 4309-4332.

Zhang, Z., Liu, B., Huang, Y., Hwang, K., Gao, H., 2010a. Mechanical properties of unidirectional nanocomposites with non-uniformly or randomly staggered platelet distribution. Journal of the Mechanics and Physics of Solids 58 (10), 1646-1660.

Zhang, Z., Zhang, Y.-W., Gao, H., 2010b. On optimal hierarchy of load-bearing biological materials. Proceedings of the Royal Society B: Biological Sciences, rspb20101093. 